\title{
A General Class of Cut-Off Model Field Theories
}

\author{
ARTHUR M. JAFFE* \\ Lyman Laboratory of Physics, Harvard University, Cambridge, Massachusetts
}

OSCAR E. LANFORD III

Department of Mathematics, University of California, Berkeley

ARTHUR S. WightMAN ${ }^{\star \star}$

Institut des Hautes Etudes Scientifiques, Bures-sur-Yvette, Essonne

Received June 5, 1969

\begin{abstract}
We show that Heisenberg picture fields and their vacuum expectation values exist for a wide class of cut-off interactions among fermions and bosons.
\end{abstract}

\section{Introduction}

The quantum field theories studied in the present paper include cutoff versions of many standard relativistic quantum field theories. They have some interest of their own as examples of non-trivial dynamics. However, the main point of studying them is to obtain information about the relativistic theories that are their putative limits as the cutoffs are removed. For this purpose, it is desirable to show

1) that the knowledge of a suitable set of matrix elements of the Hamiltonian of the cutoff theory uniquely determines the one parameter group $e^{i H t},-\infty<t<\infty$, describing the time evolution of the system,

2) that $H$ has a reasonable spectrum,

3) that the Green's functions of the theory are uniquely determined.

The results of the present paper partially satisfy these requirements. It is shown that for the models considered

$\left.1^{\prime}\right)$ there is a dense set, $D_{0}$, of vectors in the Hilbert space of states in which the Hamiltonian is essentially self-adjoint.

$2^{\prime}$ ) that $H$ has a purely discrete spectrum with finite multiplicity, bounded below and is such that its eigen functions lie in $D_{0}$.

$3^{\prime}$ ) that $D_{0}$ is invariant under the smeared fields and that for certain values of the coupling constants the ground state is non-degenerate.

* Alfred P. Sloan Foundation Fellow.

$\star \star$ On leave from Princeton University. 
$1^{\prime}$ ) completely settles 1 ). $2^{\prime}$ ) settles 2 ), $3^{\prime}$ ) does not completely settle 3 ); when the ground state is degenerate the definition of the Green's functions is ambiguous. For a class of models involving only Bose fields it is shown that the ground state is non-degenerate. On the other hand, a counter-example is given in which Fermions are present and the ground state is degenerate.

These results generalize those obtained by two of the present authors for a self-interacting Bose field [1] and for a Yukawa interaction of a spinor and a Bose field [2].

The paper deals with strong cutoffs in which only a finite number of boson modes are coupled. The vacuum expectation values are shown to be continuous in the times so that the Green's functions are unambiguously defined when the ground state is non-degenerate.

The essential mathematical idea of the proofs can be illustrated on the anharmonic oscillator

$$
H=-\frac{d^{2}}{d x^{2}}+\alpha x^{2}+\beta x^{4}, \quad \alpha \text { real, } \beta>0 .
$$

One treats $\alpha x^{2}$ as a perturbation on $-\frac{d^{2}}{d x^{2}}+\beta x^{4}$. This puts no restriction on $\alpha$ because, whatever the size of $\alpha, \alpha x^{2}$ is infinitely small compared to $-\frac{d^{2}}{d x^{2}}+\beta x^{4}$ in the sense of $\mathrm{T}$. Kato. The class of models considered is restricted by the requirement that appropriate formally positive dominating boson self-couplings (analogues of $\beta x^{4}$ in the anharmonic oscillator) be present.

Most of the remaining technical difficulties of the paper arise because of the necessity of treating not only the Hamiltonian $H$ but all its powers, in order to establish the properties of the invariant domain of vectors, $D_{0}$, and from the fact that we want to allow different species of bosons to have different order dominant self-interactions. The basic idea of the proof is to show that for every positive integer $n$

$$
\left(H_{0}^{f}+H_{1}+H_{2}\right)^{n}-\left(H_{0}^{f}+H_{1}\right)^{n}
$$

is infinitely small compared

$$
\left(H_{0}^{f}+H_{1}\right)^{n}
$$

in the sense of T. Kato. Here $H_{0}^{f}$ is the free Hamiltonian of the fermion fields: $H_{1}$ is the free Hamiltonian of the boson fields plus the dominant self-interactions and $\mathrm{H}_{2}$ is the rest of the interaction. When one expands (1.1) by the binomial theorem and estimates the resulting terms relative to $\left(H_{0}^{f}+H_{1}\right)^{n}$ one arrives at the detailed conditions on the interaction stated in the next section. 


\section{Notation and Preliminaries}

If $B$ is an operator, $D(B)$ denotes its domain and $C^{\infty}(B)=\bigcap_{n=1}^{\infty} D\left(B^{n}\right) .(B)^{-}$ denotes the closure of $B$. If $B$ and $C$ are operators, then

$$
\operatorname{Ad} C(B)=[C, B]
$$

defines an operator. With this notation the multiple commutator $[C,[C, \ldots[C, B]]]$ with $n C^{\prime} s$ is $(\operatorname{Ad} C)^{n}(B)$.

We put all fields in a space box of volume $V$ with periodic boundary conditions (3-torus!). In the strongly cutoff case, boson fields have a sharp ultra-violet cutoff. For example, if $\phi$ is a scalar boson field, the interaction term would depend on the cutoff field

$\phi_{K, V}(\boldsymbol{x})=\frac{1}{(2 V)^{1 / 2}} \sum_{\boldsymbol{k} \in \Gamma_{K, V}} \exp [-i \boldsymbol{k} \boldsymbol{x}]\left\{a_{V}^{*}(\boldsymbol{k})+a_{V}(-\boldsymbol{k})\right\} \omega(\boldsymbol{k})^{-1 / 2}$,

where

$\omega(\boldsymbol{k})=\left[\boldsymbol{k}^{2}+m^{2}\right]^{1 / 2}, \Gamma_{V}=\left\{\boldsymbol{k}: \boldsymbol{k}=\frac{2 \pi}{V^{1 / 3}} \boldsymbol{v}, \boldsymbol{v} \in Z^{3}\right\}, \Gamma_{K, V}=\Gamma_{V} \cap\{\boldsymbol{k}:|\boldsymbol{k}| \leqq K\}$,

and the $a_{V}(\boldsymbol{k}), a_{V}^{*}(\boldsymbol{k})$ are the standard Bose annihilation and creation operators normalized so that

$$
\left[a_{V}(\boldsymbol{k}), a_{V}^{*}(\boldsymbol{l})\right]_{-}=\delta_{\boldsymbol{k} \boldsymbol{l}} .
$$

Analogous formulae hold for boson fields of other tensor characters. The fermion fields will be assumed to enter the interaction in regularized form

$$
\psi_{\varrho}(\boldsymbol{x})=\int \varrho(\boldsymbol{x}-\boldsymbol{y}) d \boldsymbol{y} \psi(\boldsymbol{y})
$$

where $\varrho$ is a smooth function of fast decrease i.e. belongs to the Schwartz space $\mathscr{S}$. In contrast to the case of boson fields $\psi_{\varrho}(\boldsymbol{x})$ is a bounded operator for each $\boldsymbol{x}$.

The Hamiltonian is assumed to be a sum of a free Hamiltonian, $H_{0, V}$, and an interaction Hamiltonian, $H_{I, V}$. The different fields contribute additively to the free Hamiltonian. The contribution of a boson field like those described above is

$$
\sum_{\boldsymbol{k} \in \Gamma_{V}} a_{V}^{*}(\boldsymbol{k}) a_{V}(\boldsymbol{k}) \omega(\boldsymbol{k})
$$

the contribution of a species of fermion to $H_{0, V}$ has precisely this form but the $a_{V}(\boldsymbol{k})$ and $a_{V}^{*}(\boldsymbol{k})$ are solutions of the anti-commutation relations instead of (2.2). 
The interaction Hamiltonian is an integral over the box of an interaction Hamiltonian density:

$$
H_{I, V}=\int_{V} d \boldsymbol{x} \mathfrak{H}_{I, V}(\boldsymbol{x}) .
$$

$\mathfrak{S}_{I, V}(\boldsymbol{x})$ is a formally hermitean polynomial in the cutoff boson fields. For example, when a single hermitean scalar boson field is present

$$
\mathfrak{S}_{I, V}(\boldsymbol{x})=J^{(0)}(\boldsymbol{x})+\sum_{\alpha=1}^{2 n} J^{(\alpha)}(x) \phi_{K, V}(\boldsymbol{x})^{\alpha}
$$

where the $J^{(\alpha)}(x)$ are polynomial expressions in the fermion fields. When $N$ cutoff hermitean scalar boson fields are present

$$
\mathfrak{H}_{I, V}(\boldsymbol{x})=J^{(0)}(\boldsymbol{x})+\sum_{\alpha} J^{(\alpha)}(\boldsymbol{x}) \prod_{j=1}^{N}\left(\phi_{K, V}^{(j)}(\boldsymbol{x})\right)^{\alpha_{J}}
$$

Here $\alpha=\left(\alpha_{1}, \alpha_{2}, \ldots \alpha_{n}\right)$ is a multi-index, and the summation is taken over all multi-indices for which $0 \leqq \alpha_{\jmath} \leqq 2 n_{j}$. For convenience, the $J^{(\alpha)}$ will be referred to as fermion currents.

Now we come to the restrictions on the interaction Hamiltonian imposed by the requirement already mentioned above, that it should be dominated by boson self-interaction terms. In the case (2.6) of a single scalar boson field, it is fairly natural that that should be taken to mean

$$
J^{(2 n)}(x)=\lambda 1
$$

$\lambda$ a real number $>0$. For the case of several boson fields the natural requirement is not obvious. It turns out to be sufficient to assume

a) that the only term in (2.7) in which $\phi_{K, V}^{(j)}(x)$ occurs with maximal degree $2 n_{j}$ is of the form

$$
\lambda_{j}\left(\phi_{K, V}^{(j)}(\boldsymbol{x})\right)^{2 n_{j}}, \quad \lambda_{j}>0, j=1, \ldots N .
$$

b) For each remaining interaction term

$$
\sum_{j=1}^{N} \frac{\alpha_{j}}{2 n_{j}}<1
$$

In addition, it turns out that we need a boundedness property of the fermion currents.

c) For all $\alpha$ occuring in $\mathfrak{H}_{I, V}$

$$
\left\|\left(\operatorname{ad} H_{0, V}\right)^{n}\left(J^{(\alpha)}(x)\right)\right\| \leqq M_{n, \alpha}<\infty
$$

for some constants $M_{n, \alpha}$ and all $n=0,1,2, \ldots$

Evidently, for $N=1, a$ ) and b) reduce to the above mentioned restriction for a single boson field. As they stand these requirements exclude 
the possibility that the interaction is only linear in a boson field. We admit this possibility with the special rule that $n_{j}$ is to be set equal to 1 in this case. The reason for this rule is that there are always quadratic terms in the free boson Hamiltonian.

Conditions a), b) and c) give one precise form of the idea of dominant boson self interaction but by no means the only one. For example, it would also suffice to replace a) and b) by

$\left.a^{\prime}\right)$ that there is a term of highest degree $2 n$ in the boson fields of the form

$$
\lambda\left[\sum_{J=1}^{N}\left(\phi_{K, V}^{(j)}(\boldsymbol{x})\right)^{2}\right]^{n}
$$

$\left.b^{\prime}\right)$ that all remaining terms in the interaction Hamiltonian are of the form indicated in (2.7) with

$$
\sum_{j=1}^{N} \frac{\alpha_{j}}{2 n}<1
$$

There are cases covered by a') b') not included under a), b). For example, the cross term $\phi^{(1) 2} \phi^{(2) 2}$ which arises from $\left(\phi^{(1) 2}+\phi^{(2) 2}\right)^{2}$ is not admissible under b) because $\alpha_{1}=\alpha_{2}=2, n_{1}=n_{2}=2$ so $\sum_{j=1}^{2} \frac{\alpha_{i}}{2 n_{j}}=1$. The reader will be able to invent still other sufficient conditions and also to generalize to an arbitrary set of tensor fields after having read the next section. We content ourselves with listing some examples

Examples of Admissible Interaction Hamiltonian Densities $\mathfrak{H}_{I, V}(\boldsymbol{x})$

1) Neutral pseudo-scalar meson theory

$$
g \psi_{\varrho}^{*}(\boldsymbol{x}) \gamma^{5} \psi_{\varrho}(\boldsymbol{x}) \phi_{K, V}(\boldsymbol{x})+\lambda\left(\phi_{K, V}(\boldsymbol{x})\right)^{4}, \quad \lambda>0 .
$$

2) The $\sigma$-model of pions

$$
g\left(\sigma_{K, V}^{2}(\boldsymbol{x})+\boldsymbol{\phi}_{K, V}(\boldsymbol{x})^{2}\right)^{2}+g_{1} \boldsymbol{\phi}_{K, V}(\boldsymbol{x})^{2} \sigma_{K, V}(\boldsymbol{x})+g_{2} \sigma_{K, V}(\boldsymbol{x}), \quad g>0 .
$$

Here $\phi_{K, V}$ has three components (isospin).

3) Two hermitean scalar boson fields $\phi^{(1)}, \phi^{(2)}$

$$
g\left[\phi_{K, V}^{(1)}(x)\right]^{2} \phi_{K, V}^{(2)}(x)+\lambda\left(\phi_{K, V}^{(1)}(x)\right)^{6}, \quad \lambda>0 .
$$

Here the condition (2.9) is very restrictive. Replacing the sixth power by the fourth would not yield an admissible interaction as long as $g \neq 0$.

4) Quantum Electrodynamics of spin $\frac{1}{2}$ particles in the Coulomb gauge

$$
e \psi_{\varrho}^{*}(\boldsymbol{x}) \psi_{\varrho}(\boldsymbol{x}) \int_{V} d \boldsymbol{y} \mathscr{D}_{V}(\boldsymbol{x}-\boldsymbol{y}) \psi_{\varrho}^{*}(\boldsymbol{y}) \psi_{\varrho}(\boldsymbol{y})-e \psi_{\varrho}^{*}(\boldsymbol{x}) \boldsymbol{\alpha} \psi_{\varrho}(\boldsymbol{x}) \cdot \boldsymbol{A}_{K, V}(\boldsymbol{x})
$$


Here $\boldsymbol{A}_{K, V}(\boldsymbol{x})$ is constructed so as to satisfy $\boldsymbol{\nabla} \cdot \boldsymbol{A}_{K, V}(\boldsymbol{x})=0$, and $\mathscr{D}_{V}(\boldsymbol{x}-\boldsymbol{y})$ is the analogue on the torus, $V$, of the Coulomb potential.

It is also worth pointing out that there are physically interesting cases to which our methods in their present form do not apply. For example, in the theory of Yang-Mills fields there occur a pair of three component boson fields $\boldsymbol{g}$ and $\boldsymbol{h}$ with a dominant coupling term

$$
\lambda(\boldsymbol{g} \times \boldsymbol{h})^{2}, \quad \lambda>0 .
$$

In the "direction" $\boldsymbol{g} \times \boldsymbol{h}$ this does not grow, and therefore it does not come under the present theory. It is in fact rather unstable since a term $-\varepsilon^{2}\left(\boldsymbol{g}^{2}+\boldsymbol{h}^{2}\right)$ can make the interaction unbounded below, no matter how small $\varepsilon$ is. It would be of some interest to extend our results to this case with positivity imposed on lower order terms.

The preceding statements about the Hamiltonians of the models under discussion have been put in a form in which it is obvious what relativistic theories one might hope to obtain by removal of the cutoffs. For the calculations that follow it is much more convenient to have the boson Hamiltonians in the form of partial differential operators. This is done by introducing appropriate hermitean linear combinations $Q_{\boldsymbol{k}}^{(j)}, P_{\boldsymbol{k}}^{(j)}, j=1, \ldots N, \boldsymbol{k} \in \Gamma_{K V}$, of the annihilation and creation operators occuring in equation (2.1) so chosen that they satisfy the canonical commutation relations. The cutoff field $\phi_{K, V}^{(j)}(\boldsymbol{x})$ is then a finite linear combination of the $Q_{k}^{(j)}, \boldsymbol{k} \in \Gamma_{K, V}$ alone. The free Hamiltonian of the boson fields splits into two parts, one involving the uncoupled modes, the other, the coupled modes. The part involving the coupled modes together with the dominant self-interaction can be written as a partial differential operator. Altogether, we have

$$
H_{K, V}=H_{0}^{f}+H_{1}+H_{2}
$$

where $H_{0}^{f}$ is the free fermion Hamiltonian,

and

$$
\begin{aligned}
H_{1} & =\sum_{j=1}^{N} \sum_{\substack{\text { uncoupled } \\
\text { modes of } \phi^{(j)}}} \omega_{j}(\boldsymbol{k}) a^{(j) *}(\boldsymbol{k}) a^{(j)}(\boldsymbol{k}) \\
& +\sum_{j=1}^{N} \sum_{\substack{\text { coupled } \\
\text { modes of } \phi^{(j)}}}\left[(-\Delta)_{Q^{(j)}}+V^{(j)}\left(Q^{(j)}\right)\right],
\end{aligned}
$$

$$
H_{2}=\sum_{j=1}^{N} \sum_{\alpha} M^{(j) \alpha} \Pi\left(Q^{(j)}\right)^{\alpha} .
$$


The product in the expression for $\mathrm{H}_{2}$ runs over all coupled modes. The $M^{(j) \alpha}$ are bounded operators acting on the fermion variables; they are integrals over the box of the product of fermion currents with the sines and cosines occuring in the expansion of the $\phi^{(j)}$ in terms of $Q^{(j)}$.

\section{Strongly Cut-Off Theories}

Our first main result is

Theorem 3.1. Let $H_{K, V}=H_{0}^{f}+H_{1}+H_{2}$ where $H_{0}^{f}$ is the free field Hamiltonian of the fermions $H_{1}$ is the free field boson Hamiltonian plus the cutoff dominant boson self-interactions and $\mathrm{H}_{2}$ is the rest of the strongly cuttoff interaction as given by (2.21). $H_{0, V}$ is then the sum of the free field Hamiltonians for bosons and fermions. Let $n$ be any positive integer. Then

a) $H_{K, V}^{n}$ is essentially self-adjoint on

$$
D_{0}=C^{\infty}\left(H_{0, V}\right)
$$

b) $D\left(H_{K, V}^{n}\right)=D\left(\left(H_{0}^{f}+H_{1}\right)^{n}\right)$

c) $C^{\infty}\left(H_{K, V}\right)=C^{\infty}\left(H_{0, V}\right)$

d) The spectrum of $H_{K, V}$ is bounded below and consists of isolated eigen values with finite multiplicity.

e) Let $A(f)$ be one of the fields at $t=0$ and $A(f, t)=\operatorname{expi} H_{K, V} t A(f)$ $\exp -i H_{K, V} t$. Then for any vector $\Omega \in D_{0}$, and all positive integers $k$

$$
\frac{d^{k}}{d t^{k}} A(f, t) \Omega \in D_{0} .
$$

The proof of the theorem will be arrived at in stages. The first is a general Hilbert space argument which will later enable us to pass from the essential self-adjointness of $\left(H_{0}^{f}\right)$ and $\left(H_{1}\right)^{n}$ on their respective domains to the essential self-adjointness of $\left(H_{0}^{f}+H_{1}\right)^{n}$.

Lemma 3.2. Let $A_{1}$ and $A_{2}$ be hermitean operators in Hilbert spaces $\mathscr{H}_{1}$ and $\mathscr{H}_{2}$ respectively. Suppose that for some integer $n \geqq 1, A_{1}^{n}$ and $A_{2}^{n}$ are essentially self-adjoint on domains $D_{1}$ and $D_{2}$ respectively. Then $C=\left(A_{1} \otimes 1+1 \otimes A_{2}\right)^{j}$ is essentially self-adjoint on $D_{1} \otimes D_{2}$ for $1 \leqq j \leqq n$.

Remarks. 1) By convention, $D_{1} \otimes D_{2}$ stands for the (algebraic) tensor product of $D_{1}$ and $D_{2}$, i.e. the subset of the full tensor product $\mathscr{H}_{1} \otimes \mathscr{H}_{2}$ consisting of finite linear combinations of vectors of the form $v_{1} \otimes v_{2}$ with $v_{1} \in D_{1}, v_{2} \in D_{2}$.

2) The lemma has an easy generalization to the case of $k$ hermitean operators $A_{1} \ldots A_{k}$ acting on domains $D_{1} \ldots D_{k}$ contained respectively in Hilbert spaces $\mathscr{H}_{1} \ldots \mathscr{H}_{k}$. From the hypothesis $A_{1}^{n}, \ldots, A_{k}^{n}$ essentially 
self-adjoint for some positive integer $n$, one concludes

$$
C=\left(A_{1} \otimes 1 \otimes \cdots \otimes 1+1 \otimes A_{2} \otimes \cdots \otimes 1+\cdots 1 \otimes \cdots \otimes A_{n}\right)^{j}
$$

is essentially self-adjoint on $D_{1} \otimes \cdots \otimes D_{k}$ for $1 \leqq j \leqq n$. We give the proof for the case $k=2$ for simplicity. We will have occasion to use the case $k=3$ also.

3) The hypotheses of the lemma do not include the requirement that $A_{1}$ and $A_{2}$ be essentially self-adjoint. That property follows from the following elementary argument. Suppose $A$ is a hermitean operator and that for some integer $n \geqq 2$, at least one of the deficiency indices of $A^{n}$ is zero. Then, $A^{n}-i$, say, has dense range. Now if $a_{k}$ are the $n^{t h}$ roots of $i$, the polynomial identity $z^{n}-i=\prod_{k=1}^{n}\left(z-a_{k}\right)$ holds and therefore for each integer $k, 1 \leqq k \leqq n$ :

$$
A^{n}-i=\left(A-a_{k}\right) \prod_{j \neq k}\left(A-a_{j}\right)
$$

This shows that $A-a_{k}$ has dense range. Since for $n \geqq 2$ at least one of the $a_{k}$ is in the upper half-plane and at least one is in the lower, $A$ is essentially self-adjoint. Thus in the proof of the lemma we may assume $A_{1}$ and $A_{2}$ essentially self-adjoint.

Proof. We will prove first that

$$
D\left(\left[\left(A_{1} \otimes 1+1 \otimes A_{2}\right)^{j}\right]^{-}\right) \supset D\left(\left(A_{1}^{n}\right)^{-}\right) \otimes D\left(\left(A_{2}^{n}\right)^{-}\right) .
$$

To this end, let $\Phi$ and $\Psi$ be any two elements of $D\left(\left(A_{1}^{n}\right)^{-}\right)$and $D\left(\left(A_{2}^{n}\right)^{-}\right)$ respectively. Then there exist sequences $\Phi_{k} \in D\left(A_{1}^{n}\right)$ and $\psi_{k} \in D\left(A_{2}^{n}\right)$ such that $\Phi_{k} \rightarrow \Phi, A_{1}^{n} \Phi_{k} \rightarrow\left(A_{1}^{n}\right)^{-} \Phi, \Psi_{k} \rightarrow \Psi$, and $A_{2}^{n} \Psi_{k} \rightarrow\left(A_{2}^{n}\right)^{-} \Psi$. If $\hat{A}_{1}$ and $\hat{A}_{2}$ are respectively the self-adjoint extensions of $A_{1}$ and $A_{2}, \hat{A}_{1}^{n}$ (resp. $\hat{A}_{2}^{n}$ ) is a self-adjoint extension of $A_{1}^{n}$ (resp. $A_{2}^{n}$ ) and therefore $\hat{A}_{1}^{n}=\left(A_{1}^{n}\right)^{-}$and $\hat{A}_{2}^{n}=\left(A_{2}^{n}\right)^{-}$. Now for $0 \leqq j \leqq n$

$$
\left\|\hat{A}_{1}^{j} \chi\right\| \leqq\left\|\hat{A}_{1}^{n} \chi\right\|+\|\chi\|
$$

for all $\chi \in D\left(\left(A_{1}^{n}\right)^{-}\right)$and similarly for $\hat{A}_{2}^{j}$. (This is a consequence of the inequality $\lambda^{2 j} \leqq \lambda^{2 n}+1$, which obviously holds for all real $\lambda, 1 \leqq j \leqq n$, and the fact that by diagonalizing $\hat{A}_{1}$, one can convert (3.2) into the equivalent form

$$
\left.\left[\int \lambda^{2 j} \mu(d \lambda)\right]^{1 / 2} \leqq\left[\int\left(\lambda^{2 n}+1\right) \mu(d \lambda)\right]^{1 / 2} \leqq\left[\int \lambda^{2 n} \mu(d \lambda)\right]^{1 / 2}+\left[\int \mu(d \lambda)\right]^{1 / 2} .\right)
$$

Since $\left\|\Phi_{k}-\Phi\right\| \rightarrow 0$ and $\left\|\hat{A}_{1}^{n}\left(\Phi_{k}-\Phi\right)\right\| \rightarrow 0$, we must have $\left\|\hat{A}^{j}\left(\Phi_{n}-\Phi\right)\right\| \rightarrow 0$ i.e. $\hat{A}_{1}^{j} \Phi_{n}=A_{1}^{j} \Phi_{n} \rightarrow \hat{A}_{1}^{j} \Phi$. Similarly $A_{2}^{j} \Psi_{n} \rightarrow \hat{A}_{2}^{j} \Psi$. It follows therefore that $\left(A_{1} \otimes 1+1 \otimes A_{2}\right)^{j} \Phi_{n} \otimes \Psi_{n} \rightarrow\left(\hat{A}_{1} \otimes 1+1 \otimes \hat{A}_{2}\right)^{j} \Phi \otimes \Psi$. Hence $\Phi \otimes \Psi$ $\in D\left(\left[\left(A_{1} \otimes 1+1 \otimes A_{2}\right)^{j}\right]^{-}\right)$which completes the proof of (3.1). Further- 
more, we have

$\left[\left(A_{1} \otimes 1+1 \otimes A_{2}\right)^{j}\right]^{-} \Phi \otimes \Psi=\left(\hat{A}_{1} \otimes 1+1 \otimes \hat{A}_{2}\right)^{j} \Phi \otimes \Psi \quad 1 \leqq j \leqq n$.

We can now easily show that $\left[\left(A_{1} \otimes 1+1 \otimes A_{2}\right)^{j}\right]^{-}$is self-adjoint. Choose a vector $\Phi \in D\left(\left(A_{1}^{n}\right)^{-}\right)$and a vector $\Psi \in D\left(\left(A_{2}^{n}\right)^{-}\right)$such that their supports are compact with respect to the spectral resolution of the operators $\left(A_{1}^{n}\right)^{-}$and $\left(A_{2}^{n}\right)^{-}$respectively. Then $\Phi$ is an analytic vector for $\left(A_{1}^{n}\right)^{-}, \Psi$ is an analytic vector for $\left(A_{2}^{n}\right)^{-}$and $\Phi \otimes \Psi$ is an analytic vector for $\left[\left(A_{1} \otimes 1+1 \otimes A_{2}\right)^{j}\right]^{-}$. (The last statement is clear, as in the case of (3.2) when $\hat{A}_{1}$ and $\hat{A}_{2}$ are diagonalized.) Since the linear span of vectors of the form $\Phi \otimes \Psi$ is dense, $\left[\left(A_{1} \otimes 1+1 \otimes A_{2}\right)^{j}\right]^{-}$has a dense set of analytic vectors and is therefore self-adjoint by Nelson's theorem [6]. (3.3) shows, in fact, that

$$
\left[\left(A_{1} \otimes 1+1 \otimes A_{2}\right)^{j}\right]^{-}=\left(\hat{A}_{1} \otimes 1+1 \otimes \hat{A}_{2}\right)^{j} \quad 1 \leqq j \leqq n,
$$

on the domain of the right handside. The proof is complete.

The next four lemmas yield inequalities enabling us to estimate the terms described in the introduction just after equation (1.2). It is convenient to introduce some terminology.

Definition 3.3. Let $f$ and $g$ be two complex-valued functions defined on the same set $S$. Then $f$ dominates $g$ if there exist constants $A$ and $B$ such that

$$
|g(x)| \leqq A|f(x)|+B
$$

for all $x \in S$.

$f$ strongly dominates $g$ if for every $A>0$ there is a $B>0$ such that (3.5) holds for all $x \in S$.

Each measurable function $f$ on $\mathbb{R}^{s}$ defines an operator of pointwise multiplication on some subset of $L^{2}\left(\mathbb{R}^{s} ; d x\right): \Phi \rightarrow f \Phi$ with $(f \Phi)(x)$ $=f(x) \Phi(x)$. In the four lemmas that follow all scalar products and norms are to be taken in $L^{2}\left(\mathbb{R}^{s} ; d x\right)$.

Lemma 3.4. Let $f$ and $g$ be infinitely differentiable polynomially bounded functions on $\mathbb{R}^{n}$. Suppose

1) $f$ dominates each of its derivatives,

2) $f$ dominates $g$ and every derivative of $g$.

Then for any multi-index $\sigma=\left(\sigma_{1}, \ldots, \sigma_{s}\right)$, there are constants $A, B, C, D$ such that

$$
\left\|D^{\sigma} g \Psi\right\| \leqq A\left\|(-\Delta)^{\frac{|\sigma|}{2}} f \Psi\right\|+B\left\|(-\Delta)^{\frac{|\sigma|}{2}} \Psi\right\|+C\|f \Psi\|+D\|\Psi\|
$$

for all $\Psi \in \mathscr{S}\left(\mathbb{R}^{s}\right)$, the space of infinitely differentiable functions of fast decrease. 
Moreover, if we replace 2) by

$\left.2^{\prime}\right) f$ strongly dominates $g$ and dominates every derivative of $g$, then $A$ can be taken as any strictly positive real number when $B, C, D$, are suitably chosen.

Proof. We proceed by induction on $|\sigma|$. The lemma is clearly true when $|\sigma|=0$, because, from $|g| \leqq A|f|+B$ there follows by an elementary calculation

$$
\|g \Psi\| \leqq A\|f \Psi\|+B\|\Psi\|,
$$

which is (3.6) for this case. To carry the induction from $|\sigma|-1$ to $|\sigma|$, we distinguish the cases $|\sigma|$ even and odd. For $|\sigma|$ odd, we can write $D^{\sigma}=D_{j} D^{\sigma^{\prime}}$ for some $j$ where $\left|\sigma^{\prime}\right|=|\sigma|-1$ is even. Then

$$
\begin{aligned}
& \left\|D^{\sigma} g \Psi\right\| \leqq\left\|(-\Delta)^{(|\sigma|-1) \frac{1}{2}} D_{j} g \Psi\right\| \\
& \quad \leqq\left\|g(-\Delta)^{(|\sigma|-1) \frac{1}{2}} D_{j} \Psi\right\|+\left\|\left[g,(-\Delta)^{(|\sigma|-1) \frac{1}{2}} D_{j}\right] \Psi\right\|
\end{aligned}
$$

The first term on the right hand side is

$$
\leqq C_{1}\left\|f(-\Delta)^{(|\sigma|-1) \frac{1}{2}} D_{j} \Psi\right\|+C_{2}\left\|(-\Delta)^{(|\sigma|-1) \frac{1}{2}} D_{j} \Psi\right\|
$$

by the same argument that led to (3.7). The first term on the right hand side of (3.9) in turn becomes

$$
\leqq C_{1}\left[\left\|D_{j}(-\Delta)^{(|\sigma|-1) \frac{1}{2}} f \Psi\right\|+\left\|\left[f,(-\Delta)^{(|\sigma|-1) \frac{1}{2}} D_{j}\right] \Psi\right\|\right] .
$$

Using

$$
\left\|D_{j}(-\Delta)^{|| \sigma \mid-1) \frac{1}{2}} f \Psi\right\| \leqq\left\|(-\Delta)^{|\sigma| / 2} f \Psi\right\|
$$

to estimate the first term on the right hand side of (3.10), and similarly for the second term of (3.9) we have altogether

$$
\begin{aligned}
\left\|D^{\sigma} g \Psi\right\| & \leqq C_{1}\left\|(-\Delta)^{|\sigma| / 2} f \Psi\right\|+C_{2}\left\|(-\Delta)^{|\sigma| / 2} \Psi\right\| \\
& +C_{1}\left\|\left[f,(-\Delta)^{(|\sigma|-1) \frac{1}{2}} D_{j}\right] \Psi\right\| \\
& +\left\|\left[g,(-\Delta)^{(|\sigma|-1) \frac{1}{2}} D_{j}\right] \Psi\right\| .
\end{aligned}
$$

Here the constant $C_{1}$ can be taken arbitrarily small if $2^{\prime}$ ) holds. (In the course of this argument we have several terms replaced derivatives with the appropriate powers of $-\Delta$. An elementary justification for this is obtained by passing to the Fourier transform. The required inequalities then follow from the polynomial inequalities $\left|x^{\sigma}\right| \leqq\left(\sum_{J} x_{j}^{2}\right)^{|\sigma| / 2}$ :)

We have still to deal with the commutator terms in (3.12). Here we use the fact that

$$
\left[f,(-\Delta)^{(|\sigma|-1)^{\frac{1}{2}}} D_{j}\right]=\sum_{|\tau|<|\sigma|} D^{\tau} h_{\tau}
$$


where the $h_{\tau}$ are sums of derivatives of $f$ and hence by assumption 1) dominated by $f$. We can therefore apply the induction hypothesis to show

$$
\begin{aligned}
\left\|\left[f,(-\Delta)^{(|\sigma|-1) \frac{1}{2}} D_{j}\right] \Psi\right\| \leqq \sum_{|\tau|<|\sigma|} & \left\{A_{\tau}\left\|(-\Delta)^{|\tau| / 2} f \Psi\right\|\right. \\
& \left.+B_{\tau}\left\|(-\Delta)^{|\tau| / 2} \Psi\right\|+C_{\tau}\|f \Psi\|+D_{\tau}\|\Psi\|\right\} .
\end{aligned}
$$

Now it is easy to show that

$$
\left\|(-\Delta)^{|\tau| / 2} f \Psi\right\| \leqq K\left\|(-\Delta)^{|\sigma| / 2} f \Psi\right\|+K^{\prime}\|f \Psi\|
$$

and that $K$ may be chosen as small as desired. (Again pass to the Fourier transform and use elementary inequalities for polynomials). Similarly, $\left\|(-\Delta)^{|\tau| / 2} \Psi\right\|$ is majorized by $\left\|(-\Delta)^{|\sigma| / 2} \Psi\right\|+\|\Psi\|$. Thus, we get

$$
\begin{aligned}
& \left\|\left[f,(-\Delta)^{(|\sigma|-1)^{\frac{1}{2}}} D_{j}\right] \Psi\right\| \leqq A^{\prime}\left\|(-\Delta)^{|\sigma| / 2} f \Psi\right\| \\
& +B^{\prime}\left\|(-\Delta)^{|\sigma| / 2} \Psi\right\|+C^{\prime}\|f \Psi\|+D^{\prime}\|\Psi\|
\end{aligned}
$$

where $A^{\prime}$ may be taken as small as desired. In a similar way, we get a majorization for

$$
\left\|\left[g,(-\Delta)^{(|\sigma|-1) \frac{1}{2}} D_{j}\right] \Psi\right\|
$$

whose right hand side is of the form of the right hand side of (3.15). Combining this, (3.15) and (3.12) we have an estimate of the desired form (3.6).

When $|\sigma|$ is even the induction is slightly easier because instead of (3.8) one can write

$$
\left\|D^{\sigma} g \Psi\right\| \leqq\left\|(-\Delta)^{|\sigma| / 2} g \Psi\right\| \leqq\left\|g(-\Delta)^{|\sigma| / 2} \Psi\right\|+\left\|\left[g,(-\Delta)^{|\sigma| / 2}\right] \Psi\right\|
$$

while (3.9) becomes

$$
\leqq C_{1}\left\|f(-\Delta)^{|\sigma| / 2} \Psi\right\|+C_{2}\left\|(-\Delta)^{|\sigma| / 2} \Psi\right\|
$$

and (3.10)

$$
\leqq C_{1}\left[\left\|(-\Delta)^{|\sigma| / 2} f \Psi\right\|+\left\|\left[f,(-\Delta)^{|\sigma| / 2}\right] \Psi\right\|\right],
$$

which yields directly the analogue of (3.12)

$$
\begin{aligned}
\left\|D^{\sigma} g \Psi\right\| \leqq C_{1}\left\|(-\Delta)^{|\sigma| / 2} f \Psi\right\| & +C_{2}\left\|(-\Delta)^{|\sigma| / 2} \Psi\right\| \\
& +C_{1}\left\|\left[f,(-\Delta)^{|\sigma| / 2}\right] \Psi\right\|+\left\|\left[g,(-\Delta)^{|\sigma| / 2}\right] \Psi\right\| .
\end{aligned}
$$

Because $|\sigma|$ is even, we may replace (3.13) with

$$
\left[f,(-\Delta)^{|\sigma| / 2}\right]=\sum_{|\tau|<|\sigma|} D^{\tau} h_{\tau}
$$


and the argument goes just as before. (It is just this equality which would not be valid if $|\sigma|$ were odd.) This completes the induction and the proof of the lemma.

What kinds of functions dominate all their derivatives? It is easy to see that not every polynomial does. Take, for example, $P=x_{1}^{2}-x_{2}^{2}$. It vanishes on the line $x_{1}=x_{2}$ while $D_{1} P=2 x_{1}$ grows there. Thus, it does not dominate its derivatives. On the other hand, $P=x_{1}^{2}+x_{2}^{2}+1$ is easily seen to dominate its derivatives. More generally, we have

Lemma 3.5. Let $P_{1}, \ldots P_{J}$ be polynomials respectively of degree $2 n_{1}, \ldots, 2 n_{J}$ on $\mathbb{R}^{m_{1}}, \mathbb{R}^{m_{2}}, \ldots \mathbb{R}^{m_{J}}$. Suppose each $P_{j}$ is everywhere $\geqq 1$ and that there is a constant, $\varrho>0$, such that

$$
P_{j}(x) \geqq \varrho|x|^{2 n_{j}}, \quad j=1, \ldots J .
$$

Suppose V is the polynomial on $\mathbb{R}^{m}=\mathbb{R}^{m_{1}+m_{2}+\ldots m_{J}}=\mathbb{R}^{m_{1}} \oplus \mathbb{R}^{m_{2}} \oplus \cdots \oplus \mathbb{R}^{m_{J}}$ defined by

$$
V\left(x_{1} \ldots x_{J}\right)=P_{1}\left(x_{1}\right)+\cdots+P_{J}\left(x_{J}\right) .
$$

If $\beta>0$ then $W(x)=[V(x)]^{\beta}$ strongly dominates all its derivatives.

Proof. One easily shows by induction that

$$
D^{\sigma} W(x)=\sum_{k=1}^{|\sigma|}[V(x)]^{\beta-k} Q_{k}(x)
$$

where $Q_{k}(x)$ is a product of $k$ factors each of which is a derivative of $V(x)$ of order greater than zero. Thus

$$
D^{\sigma} W(x)=W(x) \sum_{k=1}^{|\sigma|} \sum_{\tau_{1} \ldots \tau_{k}} \alpha_{k}\left(\tau_{1} \ldots \tau_{k}\right)\left(\frac{D^{\tau_{1}} V}{V}\right) \cdots\left(\frac{D^{\tau_{k}} V}{V}\right)
$$

where the $\alpha$ 's are real numbers. Now $D^{\tau_{j}} V=0$ if the multi-index $\tau_{j}$ has a non-zero value for two different groups of variables. Thus a non-zero contribution is always of the form

$$
\frac{D^{\tau_{j}} P_{k}}{V} \leqq \frac{D^{\tau_{j}} P_{k}}{\varrho \sum_{l=1}^{J}\left|x_{l}\right|^{2 n_{l}}} .
$$

Since the numerator always has degree less than $2 n_{k}$ this approaches zero as $|x| \rightarrow \infty$. Thus $\frac{D^{\tau_{j}} V}{V} \rightarrow 0$ as $|x| \rightarrow \infty$ if $\left|\tau_{j}\right| \neq 0$, and therefore $W$ strongly dominates $D^{\sigma} W$.

It is worth remarking in passing that the assumptions of this Lemma 3.5 imply $W \geqq 1$, and our principal application of Lemma 3.4 will be to 
a case in which $f=W$. The statement and proof of Lemma 3.4 can be somewhat simplified in this case by using $\|\Psi\| \leqq\|f \Psi\|$. In particular, the inequality (3.6) is replaced by

$$
\left\|D^{\sigma} g \Psi\right\| \leqq A\left\|(-\Delta)^{|\sigma| / 2} f \Psi\right\|+B\left\|(-\Delta)^{|\sigma| / 2} \Psi\right\|+C\|f \Psi\| .
$$

The fact established in Lemma 3.5, that $W$ strongly dominates all its derivatives will now be used to bound expressions of the form $\left\|(-\Delta)^{j / 2} V^{n-j / 2} \Psi\right\|^{2}$ by a multiple of $\left\|(-\Delta+V)^{n} \Psi\right\|^{2}$.

Lemma 3.6 [1]. For each positive integer $n$, there is a constant $b$ (depending on $n$ ) such that for all $\Psi \in \mathscr{S}\left(\mathbb{R}^{m}\right)$

$$
\sum_{j=0}^{2 n}\left(\begin{array}{c}
2 n \\
j
\end{array}\right)\left\|(-\Delta)^{j / 2} V^{n-j / 2} \Psi\right\|^{2} \leqq b\left\|(-\Delta+V)^{n} \Psi\right\|^{2} .
$$

Proof. Denote the left hand side of (3.20) by $\Sigma$. Since

$$
\Sigma=\sum_{j=0}^{2 n}\left(\begin{array}{c}
2 n \\
j
\end{array}\right)\left(\Psi, V^{n-j / 2}(-\Delta)^{j} V^{n-j / 2} \Psi\right)
$$

and

$$
\begin{aligned}
\left\|(-\Delta+V)^{n} \Psi\right\|^{2}= & \left(\Psi,(-\Delta+V)^{2 n} \Psi\right) \\
= & \sum_{j=1}^{2 n}\left(\begin{array}{c}
2 n \\
j
\end{array}\right)\left(\Psi, V^{n-j / 2}(-\Delta)^{j} V^{n-j / 2} \Psi\right) \\
& + \text { commutator terms, }
\end{aligned}
$$

to prove the lemma it suffices to show that the commutator terms can be majorized in absolute value by

$$
\varepsilon \sum_{j=0}^{2 n}\left(\begin{array}{c}
2 n \\
j
\end{array}\right)\left\|(-\Delta)^{j / 2} V^{n-j / 2} \Psi\right\|^{2}+d\|\Psi\|^{2}
$$

with $\varepsilon<1$ because then

$$
\left\|(-\Delta+V)^{n} \Psi\right\|^{2} \geqq(1-\varepsilon) \Sigma-d\|\Psi\|^{2}
$$

and, since $(-\Delta+V) \geqq 1$

$$
\Sigma \leqq(1-\varepsilon)^{-1}(1+d)\left\|(-\Delta+V)^{n} \Psi\right\|^{2} .
$$

In fact, we will show that $\varepsilon$ can be taken arbitrarily small for sufficiently large $d$.

The commutator terms can be expressed as a sum of expressions of the form $\left(\Psi, V_{1} D^{\tau} V_{2} \Psi\right)$ where $V_{1}, V_{2}$ are each products of $2 n-j$ factors each of which is either $V^{1 / 2}$ or a derivative of $V^{1 / 2}$, and where $|\tau|<2 j$, $1 \leqq j \leqq 2 n-1$. By Schwarz's inequality, the above expression is majorized 
in absolute value by

$\left\|D^{\tau_{1}} V_{1} \Psi\right\|\left\|D^{\tau_{2}} V_{2} \Psi\right\| \quad$ where $\quad D^{\tau}=D^{\tau_{1}} D^{\tau_{2}}, \quad$ and $\quad\left|\tau_{1}\right|<j,\left|\tau_{2}\right| \leqq j$.

This expression in turn is majorized by

$$
\frac{1}{2 \eta}\left\|D^{\tau_{1}} V_{1} \Psi\right\|^{2}+2 \eta\left\|D^{\tau_{2}} V_{2} \Psi\right\|^{2}
$$

where $\eta$ is any number $>0$. It therefore suffices to prove that there are constants $A$ and $B$ such that

$$
\left\|D^{\tau_{2}} V_{2} \Psi\right\|^{2} \leqq A \Sigma+B\|\Psi\|^{2}
$$

and that, for any $A^{\prime}>0$ there exists a $B^{\prime}$ such that

$$
\left\|D^{\tau_{1}} V_{1} \Psi\right\|^{2} \leqq A^{\prime} \Sigma+B^{\prime}\|\Psi\|
$$

for all $\Psi \in \mathscr{S}$.

Let us prove (3.24) and then indicate the changes necessary to obtain a proof of (3.23). Lemma 3.5 tells us that $V^{n-j / 2}$ majorizes all its derivatives. Furthermore, it majorizes $V_{1}$ and every derivative of $V_{1}$. (The proof of this last runs exactly parallel to that of Lemma 3.5 itself.) Now we apply Lemma 3.4 to $D^{\tau_{1}} V_{1}$ and $V^{n-j / 2}$. It asserts that there exist constants $C$, $D, E, F$ such that

$$
\begin{aligned}
\left\|D^{\tau_{1}} V_{1} \Psi\right\| & \leqq C\left\|(-\Delta)^{\left|\tau_{1}\right| / 2} V^{n-j / 2} \Psi\right\| \\
& +D\left\|(-\Delta)^{\left|\tau_{1}\right| / 2} \Psi\right\| \\
& +E\left\|V^{n-j / 2} \Psi\right\| \\
& +F\|\Psi\| .
\end{aligned}
$$

Squaring this inequality and applying $|a b| \leqq \frac{1}{2}\left(\left(\left.a\right|^{2}+\left(\left.b\right|^{2}\right)\right.\right.$ to the cross terms appearing on the right hand side we get the same inequality with new set of constants $C, D, E, F$ and \|\| everywhere replaced by \|\|$^{2}$ :

$$
\begin{aligned}
\left\|D^{\tau_{1}} V_{1} \Psi\right\|^{2} & \leqq C\left\|(-\Delta)^{\left|\tau_{1}\right| / 2} V^{n-j / 2} \Psi\right\|^{2} \\
& +D\left\|(-\Delta)^{\mid \tau_{1} / 2} \Psi\right\|^{2} \\
& +E\left\|V^{n-j / 2} \Psi\right\|^{2} \\
& +F\|\Psi\|^{2}
\end{aligned}
$$

Since $\left|\tau_{1}\right| / 2<j / 2$, for any $A^{\prime}>0$ we can find $E^{\prime}, F^{\prime}$, and $F^{\prime \prime}$ such that $\left\|(-\Delta)^{\left|\tau_{1}\right| / 2} V^{n-j / 2} \Psi\right\|^{2} \leqq A^{\prime}\left\|(-\Delta)^{j / 2} V^{n-j / 2} \Psi\right\|^{2}+E^{\prime}\left\|V^{n-j / 2} \Psi\right\|^{2}$

$$
\begin{gathered}
\left\|(-\Delta)^{\left|\tau_{1}\right| / 2} \Psi\right\|^{2} \leqq A^{\prime}\left\|(-\Delta)^{n} \Psi\right\|^{2}+F^{\prime}\|\Psi\|^{2} \\
\left(E+E^{\prime}\right)\left\|V^{n-j / 2} \Psi\right\|^{2} \leqq A^{\prime}\left\|V^{n} \Psi\right\|^{2}+F^{\prime \prime}\|\Psi\|^{2}
\end{gathered}
$$


Therefore

$$
\begin{aligned}
\left\|D^{\tau_{1}} V_{1} \Psi\right\|^{2} & \leqq A^{\prime}\left\{\left\|(-\Delta)^{j / 2} V^{n-j / 2} \Psi\right\|^{2}\right. \\
& \left.+\left\|(-\Delta)^{n} \Psi\right\|^{2}+\left\|V^{n} \Psi\right\|^{2}\right\} \\
& +\left(F+F^{\prime}+F^{\prime \prime}\right)\|\Psi\|^{2} \\
& \leqq A^{\prime} \Sigma+\left(F+F^{\prime}+F^{\prime \prime}\right)\|\Psi\|^{2}
\end{aligned}
$$

The proof of (3.23) differs only in that because $\left|\tau_{2}\right| \leqq j, A^{\prime}$ cannot necessarily be taken arbitrarily small in (3.26), (3.27), and (3.28).

Lemma 3.7. Let $\sigma=\left(\sigma_{1} \ldots \sigma_{s}\right)$ and $\tau=\left(\tau_{1} \ldots \tau_{s}\right)$ be multi-indices. Let $x^{\tau}$ be a monomial on $\mathbb{R}^{s}=\mathbb{R}^{s_{1}} \oplus \mathbb{R}^{s_{2}} \oplus \ldots \mathbb{R}^{s_{J}}$, whose degree in the variables of $\mathbb{R}^{s_{j}}$ is $\alpha_{j}, l_{j}=1, \ldots, J$.

Suppose

$$
\frac{|\sigma|}{2}+\sum_{j=1}^{J} \frac{\alpha_{j}}{2 n_{j}}<n
$$

for some fixed integer $n$.

Then the operator $D^{\sigma} x^{\tau}$ regarded as defined on $\mathscr{S}\left(\mathbb{R}^{s}\right)$ is infinitely small in the sense of $T$. Kato with respect to $(-\Delta+V)^{n}, V$ being defined by (3.18).

Proof. The first step will be to prove that $x^{\tau}$ is strongly dominated by $V^{n-\frac{|\sigma|}{2}}$ whenever the condition (3.29) holds. In fact, if we set $r=\left[\sum_{j=1}^{N}\left|x_{j}\right|^{2 n_{j}}\right]^{1 / 2},\left|x_{j}\right|$ being the norm in $\mathbb{R}^{s_{j}}$ we have by assumption $V(x) \geqq$ const $r^{2}$ and because the individual components $x_{j}$, belonging to $\mathbb{R}^{s_{J}}$ satisfy $\left|x_{j}\right| \leqq r^{1 / n_{j}}$ we have also $\left|x^{\tau}\right| \leqq r^{\sum_{j=1}^{J}\left(\alpha_{j} / n_{j}\right)}$. Thus as $|x| \rightarrow \infty,\left|x^{\tau}\right|$ grows at most like $r^{\sum_{j}^{J}\left(\alpha_{j} / n_{j}\right)}$ while $V^{n-\frac{|\sigma|}{2}}$ grows at least like $r^{2 n-|\sigma|}$. Therefore, $x^{\tau}[V]^{\frac{\sigma}{2}-n} \rightarrow 0$ as $|x| \rightarrow \infty$, so $x^{\tau}$ is strongly dominated by $V^{n-|\sigma| / 2}$.

Since differentiating $x^{\tau}$ simply gives a multiple of a lower power of $x$, every derivative of $x^{\tau}$ is also dominated by $V^{n-|\sigma| / 2}$.

To complete the proof we apply the preceding lemmas. Suppose $\varepsilon>0$ is given. By Lemma 3.4 there exist constants $B, C, D$ such that

$$
\begin{aligned}
\left\|D^{\sigma} x^{\tau} \Psi\right\| \leqq & \varepsilon\left\|(-\Delta)^{|\sigma| / 2} V^{n-|\sigma| / 2} \Psi\right\|+B\left\|(-\Delta)^{|\sigma| / 2} \Psi\right\| \\
& +C\left\|V^{n-|\sigma| / 2} \Psi\right\|+D\|\Psi\|
\end{aligned}
$$

for all $\Psi \in \mathscr{S}\left(\mathbb{R}^{m}\right)$. We can also evidently find $D^{\prime}$ and $D^{\prime \prime}$ such that

$$
\begin{gathered}
B\left\|(-\Delta)^{|\sigma| / 2} \Psi\right\| \leqq \varepsilon\left\|(-\Delta)^{n} \Psi\right\|+\mathrm{D}^{\prime}\|\Psi\| \\
C\left\|V^{n-|\sigma| / 2} \Psi\right\| \leqq \varepsilon\left\|V^{n} \Psi\right\|+D^{\prime \prime}\|\Psi\| .
\end{gathered}
$$


Thus,

$$
\begin{aligned}
\left\|D^{\sigma} x^{\tau} \Psi\right\| \leqq \varepsilon\left[\left\|(-\Delta)^{|\sigma| / 2} V^{n-|\sigma| / 2} \Psi\right\|+\left\|(-\Delta)^{n} \Psi\right\|+\right. & \left.\left\|V^{n} \Psi\right\|\right] \\
& +\left(D+D^{\prime}+D^{\prime \prime}\right)\|\Psi\| .
\end{aligned}
$$

But from Lemma 3.6 it follows that there exist constants $A, D^{\prime \prime}$ such that $\left\|(-\Delta)^{|\sigma| / 2} V^{n-|\sigma| / 2} \Psi\right\|+\left\|(-\Delta)^{n} \Psi\right\|+\left\|V^{n} \Psi\right\| \leqq A\left\|(-\Delta+V)^{n} \Psi\right\|+D^{\prime \prime \prime}\|\Psi\|$ thus,

$$
\left\|D^{\sigma} x^{\tau} \Psi\right\| \leqq \varepsilon\left\|(-\Delta+N)^{n} \Psi\right\|+\left(D+D^{\prime}+D^{\prime \prime}+\varepsilon D^{\prime \prime \prime}\right)\|\Psi\| \text {. }
$$

Since $\varepsilon$ is any positive number and, the choice of $A$ does not depend on $\varepsilon$, this proves the lemma.

This completes the proof of the preliminary lemmas. Their application is based on a theorem of Kato.

Theorem 3.8 (Kato) Let $A$ be a linear operator on a Hilbert space $\mathscr{H}$. Suppose that $A$ is essentially self-adjoint on the domain $D(A)$ and $B$ is a hermitean operator such that

a) $D(B) \supset D(A)$.

b) For each $\varepsilon>0$ there exists a $b$ such that

$$
\|B \Phi\| \leqq \varepsilon\|A \Phi\|+b\|\Phi\|
$$

for all $\Phi \in D(A)$. Then the closures $B^{-}$and $A^{-}$satisfy

$$
\left\|B^{-} \Phi\right\| \leqq \varepsilon\left\|A^{-} \Phi\right\|+b\|\Phi\|
$$

for all $\Phi \in D\left(A^{-}\right)$. Furthermore, $A^{-}+B^{-}$is self-adjoint and

$$
D\left(A^{-}+B^{-}\right)=D\left(A^{-}\right) .
$$

(For a proof see [3], Chapter V, §4.)

For the application to the present case, we follow a line of argument similar to that developed for the $\lambda \phi^{4}$ theory in [1], in an elegant form due to J. Cannon [4].

For brevity, we introduce the following notation. If $A$ and $B$ are two linear operators, $B<A$ if $D(A) \subset D(B)$ and (3.30) holds for all $\Phi \in D(A)$. With this notation we have the following two lemmas.

Lemma 3.9 (Cannon). The set of all $B$ such that $B<A$ is a complex vector space

$$
A<A^{2} \quad \text { implies } A<A^{2}<A^{3}<\cdots .
$$

If $A$ is self-adjoint $A<A^{2}$.

Lemma 3.10 (Cannon). Suppose that for some operator $B$

$$
(\operatorname{Ad} A)^{k}(B)<A^{k+1} \quad k=0,1, \ldots
$$


Then if $l_{1}, l_{2}, \ldots$ and $l_{1}^{\prime}, l_{2}^{\prime}, \ldots$ are non-negative integers such that $\sum l_{i}+\sum l_{i}^{\prime}<l$,

$$
A^{l_{1}} B^{l_{1}^{\prime}} A^{l_{2}} B^{l_{2}} \cdots<A^{l}
$$

(For proofs, see [4]).

Proof of Theorem 3.1. We take $B=H_{2}$ and $A=H_{0}^{f}+H_{1}$. Recall that $H_{0}^{f}$ is the free fermion Hamiltonian and the Hamiltonian $H_{1}$ is a sum of the free boson Hamiltonian, $H_{0}^{b}$ and the dominant boson self-coupling. For each positive integer $n,\left(H_{0}^{f}\right)^{n}$ is essentially self-adjoint on the domain $C^{\infty}\left(H_{0}^{f}\right)$ in the $\Phi o \kappa$ space of the fermions, while $H_{1}^{n}$ is essentially selfadjoint on $C^{\infty}\left(H_{0}^{b}\right)$ in the $\Phi o \kappa$ space of the bosons. The first of these statements is elementary; the second is a basic result of [1]. It follows from Lemma 3.2 that, in the notation of that lemma, $\left(H_{0}^{f} \otimes 1+1 \otimes H_{1}\right)^{n}$ is essentially self-adjoint on $C^{\infty}\left(H_{0}^{f}\right) \otimes C^{\infty}\left(H_{0}^{b}\right)$ and therefore certainly on $C^{\infty}\left(H_{0, V}\right)$ which includes it. (The $\otimes 1$ and $1 \otimes$ will be suppressed whenever it is convenient so the operator under consideration may also be denoted $\left(H_{0}^{f}+H_{1}\right)^{n}$.)

Next we write

$$
H_{K, V}^{n}=\left(H_{0}^{f}+H_{1}\right)^{n}+\left[H_{K, V}^{n}-\left(H_{0}^{f}+H_{1}\right)^{n}\right]
$$

and study the term in square brackets on the right hand side. It is a sum of monomials in $\left(H_{0}^{f}+H_{1}\right)$ and $H_{2}$ of precisely the form of the left hand side of (3.34), when $l=n$. Thus to apply Lemma 3.10, we have only to verify

$$
\left[\operatorname{Ad}\left(H_{0}^{f}+H_{1}\right)\right]^{k}\left(H_{2}\right)<\left(H_{0}^{f}+H_{1}\right)^{k+1} .
$$

At this stage, for clarity, we indicate explicitly the action of operators as tensor products $A \otimes A^{\prime} \otimes A^{\prime \prime}$, where the first factor, $A$, acts on the coupled boson modes, the second, $A^{\prime}$, on the uncoupled boson modes and the third, $A^{\prime \prime}$, on the fermion modes. With this notation $H_{1}$ is rewritten as

$$
H_{3} \otimes 1 \otimes 1+1 \otimes H_{4} \otimes 1
$$

where $\mathrm{H}_{4}$ is the free Hamiltonian of the uncoupled boson modes, $\mathrm{H}_{3}$ is the free Hamiltonian of the coupled boson modes plus the dominant boson self-interaction. Similarly, the notation, $H_{0}^{f}$, for the free fermion Hamiltonian is replaced by $\left(1 \otimes 1 \otimes H_{0}^{f}\right)$. Finally, $H_{2}$ is a sum of terms of the form $Q^{\tau} \otimes 1 \otimes F$ where $F$ is a bounded operator.

Thus $\left[\operatorname{Ad}\left(H_{0}^{f}+H_{1}\right)\right]^{k}\left(H_{2}\right)$ is a sum of terms of the form

$$
[\operatorname{Ad}(-\Delta)]^{k}\left(Q^{\tau}\right) \otimes 1 \otimes\left[\operatorname{Ad}\left(H_{0}^{f}\right)\right]^{k}(F) .
$$

By assumption (2.11), the last factor is a bounded operator, $F_{k}$. The first is a sum of terms of the form $D^{\sigma} Q^{\tau^{\prime}}$ where $\left|\tau^{\prime}\right|<|\tau|$ and $|\sigma| \leqq 2 k$. Now by 
assumption $\tau$ is such that the criterion (2.10) is satisfied. That implies $Q^{\tau}<H_{3}$ by Lemma 3.7. (Set $n=1,|\sigma|=0$ there.) Then using $|\sigma| \leqq 2 k,\left|\tau^{\prime}\right|<|\tau|$ we get that (3.29) is satisfied with $n=k+1$ so $D^{\tau^{\prime}} Q^{\sigma}<H_{3}^{k+1}$ again by Lemma 3.7. It remains only to show from this that $(3.38)<\left(H_{0}^{f}+H_{1}\right)^{k+1}$ The argument goes as follows. Vectors of $C^{\infty}\left(H_{3}\right) \otimes C^{\infty}\left(H_{4}\right) \otimes C^{\infty}\left(H_{0}^{f}\right)$ (algebraic tensor product) can be wirtten as finite sums $\chi=\sum_{i} \Phi_{i} \otimes \Psi_{i}$ where $\Phi_{i} \in C^{\infty}\left(H_{3}\right)$ and $\Psi_{i} \in C^{\infty}\left(H_{4}\right) \otimes C^{\infty}\left(H_{0}^{f}\right)$. Without loss of generality the set $\left\{\Psi_{i}\right\}$ may be assumed orthonormal. Noting $D^{\sigma^{\prime}} Q^{\tau^{\prime}} \otimes 1 \otimes F_{k}$ $=\left(1 \otimes 1 \otimes F_{k}\right)\left(D^{\sigma} Q^{\tau^{\prime}} \otimes 1 \otimes 1\right)$ and $\left\|1 \otimes 1 \otimes F_{k}\right\|=\left\|F_{k}\right\|$, we have therefore

$$
\begin{aligned}
&\left\|\left(D^{\sigma} Q^{\tau^{\prime}} \otimes 1 \otimes F_{k}\right) \chi\right\| \leqq\left\|F_{k}\right\|\left\|\sum_{i} D^{\sigma} Q^{\tau^{\prime}} \Phi_{i} \otimes \Psi_{i}\right\| \\
& \leqq\left\|F_{k}\right\|\left[\varepsilon\left\|\left(H_{3}^{k+1} \otimes 1\right)\left(\sum_{i} \Phi_{i} \otimes \Psi_{i}\right)\right\|+b\|\chi\|\right] \\
& \leqq \varepsilon\left\|F_{k}\right\|\left\|\left(H_{0}^{f}+H_{1}\right)^{k+1} \chi\right\|+b\left\|F_{k}\right\|\|\chi\| .
\end{aligned}
$$

All but the last of these steps are elementary. Putting aside its justification for a moment, one can pass from (3.39) to the desired inequality valid everywhere on the domain of $\left(H_{0}^{f}+H_{1}\right)^{k+1}$ by closure. Since $\left\|F_{k}\right\|$ is independent of $\chi$ and $\varepsilon$ can be chosen arbitrarily small, this shows (3.38) $<\left(H_{0}^{f}+H_{1}\right)^{k+1}$.

The last step of (3.39) follows from the fact that if $A$ and $B$ are essentially self-adjoint positive operators, commuting on $C^{\infty}(A) \cap C^{\infty}(B)$, then

$$
\|A \Phi\| \leqq\|(A+B) \Phi\|
$$

for all $\Phi \in C^{\infty}(A) \cap C^{\infty}(B)$. To see this one notes that under the same assumptions $A B$ is positive $\left((\Phi, A B \Phi)=\left\|A^{1 / 2} B^{1 / 2} \Phi\right\|^{2}\right)$ so

$$
\left(\Phi, A^{2} \Phi\right) \leqq\left(\Phi,\left(A^{2}+B^{2}+2 A B\right) \Phi\right) .
$$

The square root of this inequality is (3.40).

This completes the proof that all monomials appearing when $H_{K, V}^{n}-\left(H_{0}^{f}+H_{1}\right)^{n}$ is expanded satisfy the hypotheses of Lemma 3.10. Thus each such monomial is $<\left(H_{0}^{f}+H_{1}\right)^{n}$. Since, by Lemma 3.9, the operators $<\left(H_{0}^{J}+H_{1}\right)^{n}$ form a vector space, we have

$$
H_{K, V}^{n}-\left(H_{0}^{f}+H_{1}\right)^{n}<\left(H_{0}^{f}+H_{1}\right)^{n}
$$

and therefore, by Kato's Theorem 3.8, $H_{K, V}^{n}$ is essentially self-adjoint on $D\left(\left(H_{0}^{f}+H_{1}\right)^{n}\right)$ and $D\left(H_{K, V}^{n}\right)=D\left(\left(H_{0}^{f}+H_{1}\right)^{n}\right)$. This proves b) of Theorem 3.1. a) follows from Kato's Theorem 3.8 and the fact proved above that $\left(H_{0}^{f}+H_{1}\right)^{n}$ is essentially self-adjoint on $C^{\infty}\left(H_{0, V}\right)$.

c) As a consequence of b) $C^{\infty}\left(H_{K, V}\right)=C^{\infty}\left(H_{0}^{f}+H_{1}\right)$. Thus, to prove c) it suffices to establish $C^{\infty}\left(H_{0}^{f}+H_{1}\right)=C^{\infty}\left(H_{0, V}\right)$, that is, the addition 
of the dominant boson self-interaction to the free Hamiltonian $H_{0}^{f}+H_{0}^{b}$ $=H_{0, V}$ does not change the intersection of the domains of all the powers of the free Hamiltonian. Now one of the basic results of [1] was that $C^{\infty}\left(H_{1}\right)=C^{\infty}\left(H_{0}^{b}\right)$. Thus, the problem of proving c) is reduced to showing that the addition of $H_{0}^{f}$ to $H_{1}$ and $H_{0}^{b}$ does not affect this relation. That in turn is a consequence of

$$
D\left(\left(H_{0}^{f} \otimes 1+1 \otimes H_{1}\right)^{n}\right)=D\left(\left(H_{0}^{f} \otimes 1\right)^{n}\right) \cap D\left(\left(1 \otimes H_{1}\right)^{n}\right)
$$

and similarly

$$
D\left(\left(H_{0}^{f} \otimes 1+1 \otimes H_{0}^{b}\right)^{n}\right)=D\left(\left(H_{0}^{f} \otimes 1\right)^{n}\right) \cap D\left(\left(1 \otimes H_{0}^{b}\right)^{n}\right) .
$$

(We have reinstated the tensor product notation for clarity). (3.42) and (3.43) follow from the positivity of $H_{0}^{f}, H_{0}^{b}$, and $H_{1}$. That may be seen as follows. We have, surely, that the left hand sides of (3.42) and (3.43) include the right hand sides. Furthermore,

$$
D\left(\left(H_{0}^{f} \otimes 1+1 \otimes H_{1}\right)^{n}\right) \supset D\left(\left(H_{0}^{f}\right)^{n}\right) \otimes D\left(\left(H_{1}\right)^{n}\right)
$$

(again algebraic tensor product). On vectors velonging to the right hand side

$$
\left(H_{0}^{f} \otimes 1+1 \otimes H_{1}\right)^{n}=\sum_{k=0}^{n}\left(\begin{array}{l}
n \\
k
\end{array}\right)\left(H_{0}^{f}\right)^{k} \otimes\left(H_{1}\right)^{n-k}
$$

and because all operators occuring are pusitive and commute

$$
\left\|\left(\begin{array}{l}
n \\
k
\end{array}\right)\left(H_{0}^{f}\right)^{k} \otimes\left(H_{1}\right)^{n-k}\left(\Phi_{l}-\Phi_{m}\right)\right\| \leqq\left\|\left(H_{0}^{f} \otimes 1+1 \otimes H_{1}\right)^{n}\left(\Phi_{l}-\Phi_{m}\right)\right\|
$$

for any Cauchy sequence $\left(\Phi_{i}\right)$ of vectors belonging to the right hand side of (3.44). Thus, passing to the closure, we find that $\Phi \in D\left(\left(H_{0}^{f} \otimes 1+1 \otimes H_{1}\right)^{n}\right)$ implies $\Phi \in D\left(\left(H_{0}^{f} \otimes 1\right)^{n}\right) \cap D\left(\left(1 \otimes H_{1}\right)^{n}\right)$. There is an analogous argument with $H_{1}$ replaced by $H_{0}^{b}$ so the proof of c) is complete.

d) Both $H_{0}^{f}$ and $H_{1}$ have compact resolvents $[2,1]$. Thus, their eigenvalues $E_{i}^{f}$ and $E_{j}$ respectively have finite multiplicity and cluster only to infinity. Their eigen functions $\Phi_{i}^{f}$ and $\Phi_{j}$ respectively are complete in the respective $\Phi o \kappa$ spaces. $H_{0}^{f}+H_{1}$ has eigen values $E_{i}^{f}+E_{j}$ with corresponding eigen functions $\Phi_{i}^{f} \otimes \Phi_{j}$. Since they are complete $H_{0}^{f}+H_{1}$ also has compact resolvent. Now as we have seen in the proof of a), $\mathrm{H}_{2}$ is infinitely small relative to $H_{0}^{f}+H_{1}$. It is a general result of perturbation theory that the addition of such a perturbation preserves the compactness of the resolvent. (See [3], p. 214, Theorem 3.17). Thus d) is proved.

Now we prove e). b) tells us that $C^{\infty}\left(H_{0, V}\right)$ is invariant under $H_{K, V}$ and $e^{i H_{K}, V^{t}}$. (For $H_{K, V}$ this is obvious; for $e^{i H_{K}, V^{t}}$, it is an easy consequence 
of the spectral theorem.) Since, from the definition of $A(f, t)$,

$$
\frac{d^{n}}{d t^{n}} A(f, t) \Omega=\left[i H_{K, V}, \frac{d^{n-1}}{d t^{n-1}} A(f, t)\right] \Omega,
$$

we see that to prove e) it suffices to prove that $A(f) \Omega$ is in $C^{\infty}\left(H_{0, V}\right)$ if $\Omega$ is. There are two things to verify: First that $D(A(f)) \supset C^{\infty}\left(H_{0, V}\right)$ and second that $A(f) C^{\infty}\left(H_{0, V}\right) \subset C^{\infty}\left(H_{0, V}\right)$. Both these statements require only elementary calculations in $\Phi o \kappa$ space. For all fields $A(f)$, boson or fermion, one finds that for each $\varepsilon>0$ there is a $b$ such that

$$
\|A(f) \Phi\| \leqq\left[\varepsilon\left\|H_{0, V} \Phi\right\|+b\|\Phi\|\right]|f|_{s}
$$

for all $\Phi \in D\left(H_{0, V}\right)$. Here $/\left.\right|_{s}$ is some norm on the test function space. For example, for fermion fields of spin $\frac{1}{2}$ it is the ordinary $L^{2}$ norm, while for the conjugate scalar boson field $\pi$,

$$
|f|_{s}=\left[\int|\hat{f}(\boldsymbol{p})|^{2}\left[m^{2}+p^{2}\right]^{1 / 2} d \boldsymbol{p}\right]^{1 / 2}
$$

will do. From this inequality (3.47), one gets immediately $D(A(f)) \supset D\left(H_{0, V}\right)$ and therefore a fortiori $D(A(f)) \supset C^{\infty}\left(H_{0, V}\right)$. To see the invariance of $C^{\infty}\left(H_{0, V}\right)$ under $A(f)$, note that the identity

$$
H_{0, V} A(f)=A(f) H_{0, V}+A\left(f^{\prime}\right)
$$

holds on, say, the vectors with only a finite number of non-zero components in $\Phi_{O K} \kappa$ space, all of which are infinitely differentiable and of compact support. Here $f^{\prime}$ is obtained from $f$ by multiplying its Fourier transform by kinematical factors (e.g., $\omega(\boldsymbol{p})$ to some power) that depend on which field is under consideration. From (3.47) and (3.49) one gets, passing to the closure, $A(f) D\left(H_{0, V}^{n}\right) \subset D\left(H_{0, V}^{n-1}\right)$ as desired.

Theorem 3.1 together with some of the information obtained in the course of its proof permit one to construct the basic objects, vacuum expectation values and Green's functions, in terms of which the content of a field theory is customarily expressed. Notice first that any eigenfunction $\Omega$ of $H$ is certainly in $C^{\infty}\left(H_{K, V}\right)$ and therefore any product of the fields smeared with test functions in $\mathscr{S}$ in the space variables is certainly applicable to it. Thus the expectation value

$$
\left(\Omega, \prod_{j} A_{j}\left(f_{j}, t_{j}\right) \Omega\right)
$$

is well defined. From c) we see that it is infinitely differentiable in the $t$ 's. It is clearly a multilinear functional of the $f$ 's. Theorem $3.1 \mathrm{e}$ ) implies that it is in fact a tempered distribution in each of these variables and thus by the nuclear theorem in all the space variables together. The time ordering operation carried out on the expressions (3.50) is unambigous 
because they are smooth in the times; that shows the Green's functions exist as piecewise $C^{\infty}$ functions of the times and tempered distributions of the space variables.

The preceding discussion is valid for any eigenfunction $\Omega$ of $H$. Of course, the traditional definition of vacuum expectation values and Green's functions uses the ground state for $\Omega$. It should be noticed that nothing said up to this point prevents the ground state from being degenerate. This does not cause any trouble with the existence proof for the vacuum expectation values and Green's functions; it just means that unless further physical requirements are imposed there is, in general, a family of equally admissible vacuum expectation values and Green's functions.

These general remarks can be supplemented and made more precise in special cases. When only bosons are present, the ground state is nondegenerate by virtue of the same argument that shows that the ground state of the non-relativistic Schrödinger equation for spin-less particles has no nodes. On the other hand, the following example shows that when fermions are present no such general argument can exist.

Example 3.11. Consider a single species of fermion interacting with a single species of boson. Suppose $H_{2}=g A^{*} A \tilde{Q}$ where $A^{*}$ is the creation operator for a fermion mode with corresponding contribution to the free Hamiltonian $M A^{*} A$, and $\tilde{Q}$ is some linear combination of $Q_{1} \ldots Q_{m}$. Then the Hilbert space may be written as a direct sum $\mathscr{H}=\mathscr{H}_{0} \oplus \cdot \mathscr{H}_{1}$ where $\mathscr{H}_{0}$ is the subspace on which $A^{*} A=0$ and $\mathscr{H}_{1}$ that on which $A^{*} A=1$. Each of these subspaces is mapped into itself by the full Hamiltonian $H$. On $\mathscr{H}_{0}$ the lowest eigen-value $E_{0}$ of $H$ is that of $H_{1}=-\Delta_{Q}+V(Q)$ while on $\mathscr{H}_{1}$ the lowest eigenvalue of $H$ is equal to the lowest eigenvalue of $-\Delta_{Q}+V(Q)+M+g \tilde{Q}$. By adjusting $g$ one can make this eigenvalue take any value between $E_{0}+M$ and $-\infty$, so in particular one can make it equal to $E_{0}$. With this choice of $g$ the ground state of $H$ is at least two-fold degenerate.

Using Theorem 3.1 and the above remarks it is completely straight forward to verify, following the pattern of $[1,2]$ that the standard Heisenberg equations of motion for the operators $A(f, t)$ hold as equalities on vectors of $C^{\infty}\left(H_{0, V}\right)$ and that the usual differential equations for the Green's functions hold as equations in tempered distributions. We summarize in

Theorem 3.12. For quantum field theories satisfying the hypotheses of Theorem 3.1, vacuum expectation values exist as tempered distributions in the space variables and infinitely differentiable functions of the time variables. The Green's functions are tempered distributions and piecewise infinitely differentiable in the times. The standard Heisenberg equations of motion for the field operators are valid, when smeared with a test 
function in the space variable, as operator equalities valid on the vectors of $C^{\infty}\left(H_{0, V}\right)$. The standard differential equations for the Green's functions are valid as relations between tempered distributions.

\section{References}

1. Jaffe, A. M.: Dynamics of a cut-off $\lambda \varphi^{4}$ theory. Princeton Thesis 1965 and Amer. Math. Soc. Memoir to appear.

2. Lanford, O. E.: Construction of quantum fields interacting by a cut-off Yukawa coupling. Princeton Thesis 1966 and Amer. Math. Soc. Memoir to appear.

3. Kato, T.: Perturbation theory for linear operators. Berlin-Heidelberg-New York: Springer 1966.

4. Cannon, J.: Princeton Thesis 1967. To appear.

5. See theorems 8.3 and 8.4 proved by Lanford, O., A. Jaffe, and S. Doplicher respectively quoted in Cargèse lectures 1964 edited by M. Levy Gordon and Breach N. Y. 1967. See also I. Segal: Non-linear functions of stochastic processes I. Jour. Funct. Anal. to appear.

6. Nelson, E.: Ann. of Math. 70, 572 (1959).

Arthur M. Jaffe

Lyman Laboratory of Physics

Harvard University

Cambridge, Massachusetts, USA
Oscar E. Lanford III

Department of Mathematics

University of California

Berkeley, California, USA

Arthur S. Wightman

Dept. of Physics

Princeton University

Princeton, New Jersey 08540, USA 